Draft Version March 3, 2017

Preprint typeset using $\mathrm{LAT}_{\mathrm{E}} \mathrm{X}$ style emulateapj v. 12/16/11

\title{
NORTHERN SKY GALACTIC COSMIC RAY ANISOTROPY BETWEEN 10 AND 1000 TEV WITH THE TIBET AIR SHOWER ARRAY
}

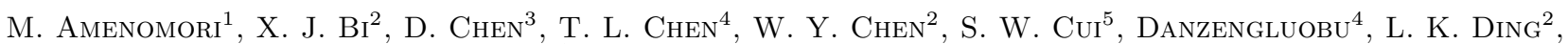
C. F. Feng ${ }^{6}$, Zhaoyang Feng ${ }^{2}$, Z. Y. Feng ${ }^{7}$, Q. B. Gou ${ }^{2}$, Y. Q. GuO ${ }^{2}$, H. H. He ${ }^{2}$, Z. T. He ${ }^{5}$, K. Hibino ${ }^{8}$, N. Hotta $^{9}$,

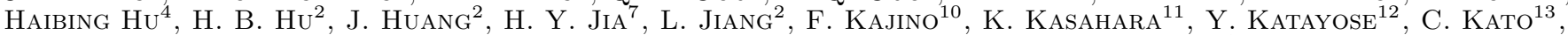

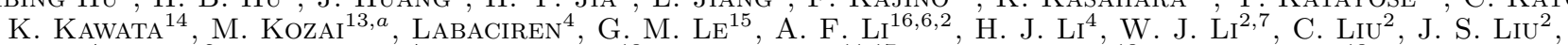
M. Y. $\operatorname{Lid}^{4}$, H. Lu ${ }^{2}$, X. R. MenG ${ }^{4}$, T. Miyazaki ${ }^{13}$, K. Mizutani ${ }^{11,17}$, K. Munakata ${ }^{13}$, T. Nakajima ${ }^{13}$, Y. Nakamura ${ }^{13}$, H. NAnjo ${ }^{1}$, M. Nishizawa ${ }^{18}$, T. Niwa ${ }^{13}$, M. Ohnishi ${ }^{14}$, I. Ohta ${ }^{19}$, S. Ozawa ${ }^{11}$, X. L. Qian ${ }^{6,2}$, X. B. Qu ${ }^{2}$, T. Saito ${ }^{20}$,' T. Y. Saito ${ }^{21}$, M. Sakata ${ }^{10}$, T. K. Sako ${ }^{14}$, J. ShaO ${ }^{2,6}$, M. Shibata ${ }^{12}$, A. Shiomi ${ }^{22}$, T. Shirai ${ }^{8}$, H. Sugimoto ${ }^{23}$,

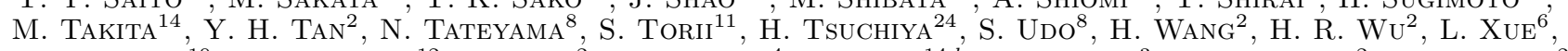
Y. Yamamoto ${ }^{10}$, K. Yamauchi ${ }^{12}$, Z. YAnG ${ }^{2}$, A. F. Yuan ${ }^{4}$, T. Yuda ${ }^{14, b}$, L. M. Zhai ${ }^{3}$, H. M. Zhang ${ }^{2}$, J. L. ZhanG $^{2}$,

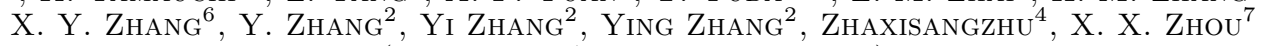
(The Tibet AS $\gamma$ Collaboration)

${ }^{1}$ Department of Physics, Hirosaki University, Hirosaki 036-8561, Japan

${ }^{2}$ Key Laboratory of Particle Astrophysics, Institute of High Energy Physics, Chinese Academy of Sciences, Beijing 100049, China

${ }^{3}$ National Astronomical Observatories, Chinese Academy of Sciences, Beijing 100012, China

${ }^{4}$ Department of Mathematics and Physics, Tibet University, Lhasa 850000, China

${ }^{5}$ Department of Physics, Hebei Normal University, Shijiazhuang 050016, China

${ }^{6}$ Department of Physics, Shandong University, Jinan 250100, China

${ }^{7}$ Institute of Modern Physics, Southwest Jiaotong University, Chengdu 610031, China

${ }^{8}$ Faculty of Engineering, Kanagawa University, Yokohama 221-8686, Japan

${ }^{9}$ Faculty of Education, Utsunomiya University, Utsunomiya 321-8505, Japan ${ }^{10}$ Department of Physics, Konan University, Kobe 658-8501, Japan

${ }^{11}$ Research Institute for Science and Engineering, Waseda University, Tokyo 169-8555, Japan

${ }^{12}$ Faculty of Engineering, Yokohama National University, Yokohama 240-8501, Japan

${ }^{13}$ Department of Physics, Shinshu University, Matsumoto 390-8621, Japan

${ }^{14}$ Institute for Cosmic Ray Research, University of Tokyo, Kashiwa 277-8582, Japan

${ }^{15}$ National Center for Space Weather, China Meteorological Administration, Beijing 100081, China

${ }^{16}$ School of Information Science and Engineering, Shandong Agriculture University, Taian 271018, China ${ }^{17}$ Saitama University, Saitama 338-8570, Japan

18 National Institute of Informatics, Tokyo 101-8430, Japan

${ }^{19}$ Sakushin Gakuin University, Utsunomiya 321-3295, Japan

${ }^{20}$ Tokyo Metropolitan College of Industrial Technology, Tokyo 116-8523, Japan

${ }^{21}$ Max-Planck-Institut für Physik, München D-80805, Deutschland

${ }^{22}$ College of Industrial Technology, Nihon University, Narashino 275-8576, Japan

${ }^{23}$ Shonan Institute of Technology, Fujisawa 251-8511, Japan

${ }^{24}$ Japan Atomic Energy Agency, Tokai-mura 319-1195, Japan

${ }^{a}$ now at: ISAS/JAXA Sagamihara 252-5210, Japan

${ }^{b}$ Deceased.

Draft version March 3, 2017

\section{ABSTRACT}

We report on the analysis of the $10-1000 \mathrm{TeV}$ large-scale sidereal anisotropy of Galactic cosmic rays (GCRs) with the data collected by the Tibet Air Shower Array from 1995 October to 2010 February. In this analysis, we improve the energy estimate and extend the decl. range down to $-30^{\circ}$. We find that the anisotropy maps above $100 \mathrm{TeV}$ are distinct from that at a multi-TeV band. The so-called tail-in and loss-cone features identified at low energies get less significant, and a new component appears at $\sim 100 \mathrm{TeV}$. The spatial distribution of the GCR intensity with an excess (7.2 $\sigma$ pre-trial, $5.2 \sigma$ post-trial) and a deficit $(-5.8 \sigma$ pre-trial) are observed in the $300 \mathrm{TeV}$ anisotropy map, in close agreement with IceCube's results at $400 \mathrm{TeV}$. Combining the Tibet results in the northern sky with IceCube's results in the southern sky, we establish a full-sky picture of the anisotropy in hundreds of $\mathrm{TeV}$ band. We further find that the amplitude of the first order anisotropy increases sharply above $\sim 100 \mathrm{TeV}$, indicating a new component of the anisotropy. All these results may shed new light on understanding the origin and propagation of GCRs.

\section{INTRODUCTION}

The arrival directions of Galactic cosmic rays (GCRs) are nearly isotropic due to deflections in the Galactic magnetic field (GMF).Only weak anisotropy is expected from the diffusion and/or drift of GCRs in GMF. Observations of ground-based air shower arrays and underground muon detectors do show the existence of small anisotropies with relative amplitudes of the order of $10^{-4}$ to $10^{-3}$ at energies from $100 \mathrm{GeV}$ to hundreds of $\mathrm{TeV}$ (see Figure 51). However, the variation of the amplitude with energy seems to be difficult to interpret in terms of the conventional GCR diffusion model in the Galaxy (e.g. (Moskalenko et al. 2002; Ahlers \& Mertsch 2016)). The study of GCR anisotropy, therefore, is important to understand the origin and propagation of GCRs.

Only a few results of the anisotropy in the energy range from hundreds of $\mathrm{TeV}$ up to $\sim 10 \mathrm{PeV}$ have been re- 
ported, primarily due to the low fluxes of cosmic rays (CRs) in this energy range. EAS-TOP collaboration reported for the first time a detection of anisotropy at $\sim 200 \mathrm{TeV}$ (Aglietta et al. 1996). With the accumulation of data, they improved their result later and reported a sharp increase of the anisotropy amplitude at primary energies around $\sim 370 \mathrm{TeV}$ (Aglietta et al. 2009). At the PeV energy region, the Akeno experiment reported an increase of the CR anisotropy amplitude in 1986 (Kifune et al. 1986). No hint of the anisotropy, on the other hand, has been found in the KASCADE data at higher energies between 0.7 and $6 \mathrm{PeV}$ (Antoni et al. 2004). Recently, the IceCube collaboration reported the anisotropy observed in the southern sky, showing a new feature different from that obtained by EAS-TOP (Abbasi et al. 2012). A clear deficit with a post-trial significance of $-6.3 \sigma$ at $400 \mathrm{TeV}$ was detected, which was then confirmed by the result from Ice-Top Aartsen et al. 2013). The Ice-Top data further revealed the existence of anisotropy at energies up to $1 \mathrm{PeV}$ (Aartsen et al. 2013).

The Tibet Air Shower (AS) array collaboration presented the first two-dimensional anisotropy measurements in an energy region from several $\mathrm{TeV}$ to several hundred $\mathrm{TeV}$. The anisotropy features, known as the "tail-in" and "loss-cone" features, were observed with very high significances (Amenomori et al. 2006). A new component anisotropy at multi-TeV energies from the Cygnus direction was also reported Amenomori et al. 2006). It has been shown that the amplitude of the first order anisotropy decreases above a few hundred $\mathrm{TeV}$, indicating the co-rotation of GCRs around the Galactic center. With more data accumulated, hints of $\sim 300$ $\mathrm{TeV}$ anisotropies have been revealed (Feng et al. 2009; Amenomori et al. 2013). The anisotropy feature was found to be different from those in lower energy regions and in agreement with IceCube's result at $400 \mathrm{TeV}$ (Abbasi et al. 2012). These analyses of Tibet AS array data cover decl. from $-15^{\circ}$ to $75^{\circ}$, yet leaving a gap to be connected with IceCube's result in the southern sky. Here we extend these analyses to include events with zenith angle up to $60^{\circ}$, which corresponds to a coverage of decl. from $-30^{\circ}$ to $90^{\circ}$ (Amenomori et al. 2015). Combining with IceCube's results, we present for the first time a full-sky anisotropy observed at hundreds of $\mathrm{TeV}$. By improving the reconstruction of primary energy, we will also extend the analyzed energy range to two decades between $10 \mathrm{TeV}$ and $1 \mathrm{PeV}$, which is also the widest coverage of such works.

\section{ANALYSIS}

\subsection{Experiment and Data reconstruction}

The Tibet AS Array is located at Yangbajing in Tibet, China $\left(90.522^{\circ} \mathrm{E}, 30.102^{\circ} \mathrm{N}, 4300 \mathrm{~m}\right.$ above sea level, 606 $\mathrm{g} / \mathrm{cm}^{2}$ atmospheric depth). The detector array consists of plastic scintillation detectors with an area of $0.5 \mathrm{~m}^{2}$ each. The effective area of the Tibet AS array has been gradually enlarged, via adding the same-type detectors to the array. The Tibet I array was constructed in 1990 , using 65 plastic scintillation detectors placed on grids with $15 \mathrm{~m}$ spacing. It was then upgraded to 221 detectors on $15 \mathrm{~m}$ grids, covering a total of $36,900 \mathrm{~m}^{2}$, known as the Tibet II array. It began operation in 1995 October, with a trigger rate of $\sim 230 \mathrm{~Hz}$. The Tibet II was then upgraded to the current Tibet III, a denser array with $7.5 \mathrm{~m}$ grids, in 1999 and 2003 (Amenomori et al. 2003). The trigger rate is $\sim 1700 \mathrm{~Hz}$ for the Tibet III array.

In order to maintain the uniformity of the array performance, we analyze the data keeping the same configuration of the Tibet II array throughout the observation period from 1995 October to 2010 February, so that the full data sample taken by Tibet II and Tibet III array can be used in the present analysis. The traditional shower reconstruction procedure is applied to get all the parameters of one shower, such as the core position, zenith, and azimuth angles $(\theta, \phi)$ of the incident direction and shower size $\sum \rho_{F T}$ (the sum of the number of particles per $\mathrm{m}^{2}$ counted by all the fast-timing [FT] detector). The following three criteria are applied to select events for further analyses: (1) each AS event should fire four or more detectors, with each recording 1.25 or more particles; (2) the AS core position should be located inside the array; and (3) zenith angle $\theta<60^{\circ}$.

\subsection{Estimation of the CR Energy}

The ASs reaching the array with a large zenith angle $\theta$ travel through a larger slant atmospheric depth than the vertical ones. This leads to a zenith angle dependence of the relation between $\sum \rho_{F T}$ and the primary particle energy. In most of the previous works of the Tibet AS $\gamma$ Collaboration (Amenomori et al.|2003, 2005a, 2006; Feng et al. 2009; Amenomori et al. 2013), the shower size $\sum \rho_{F T}$ is solely adopted to infer the primary energy of an AS without considering the zenith angle dependence. This approximation works well for small zenith angles $\left(\theta<\sim 40^{\circ}\right)$, considering the natural fluctuation in the development of the AS and the limited resolution of the primary energy.

In this work, we intend to explore the anisotropy with decl. down to $-30^{\circ}$ by including showers with zenith angles up to $60^{\circ}$. Therefore, the zenith angle dependence of the energy reconstruction must be taken into account. We develop a two-dimensional selection criterion in the $\sum \rho_{F T}-\sec \theta$ plane for the energy reconstruction.

The uncertainty of the CR energy reconstruction has been estimated with a full Monte Carlo (MC) simulation of CR interactions in the atmosphere by CORSIKA (version 6.204; (Heck et al. 1998). The hadronic interaction model QGSJET01c and the detector response modeled by Epics (version 8.65; (Kasahara 2006) are used. In this simulation, we adopt the composition and spectrum models of primary CRs given in (Hörandel 2003) as inputs.

Figure 1 shows the simulated distribution in the primary CR energy as a function of $\sum \rho_{F T}$ and $\sec \theta$. It shows that for a given range of $\sum \rho_{F T}$, small zenith angle events (at $\sec \theta \sim 1$ ) are dominated by CRs with lower average energy, compared with large zenith angle events (at $\sec \theta \sim 2$ ). We show regions of constant primary energies $(15,50,100,300$, and $1000 \mathrm{TeV})$ in the plane of $\left(\sum \rho_{F T}, \sec \theta\right)$ as regions delimited by the dashed lines in Figure 1, This grouping enables us to select events in five energy samples with minimal overlappings.

Figure 2 shows the simulated primary energy distributions of the five energy samples, as indicated by the dashed lines in Figure 1]. The uncertainty of the primary energy estimate is dominated by the fluctuation of the AS. Event numbers in five energy samples are $2.33 \times 10^{10}$ 


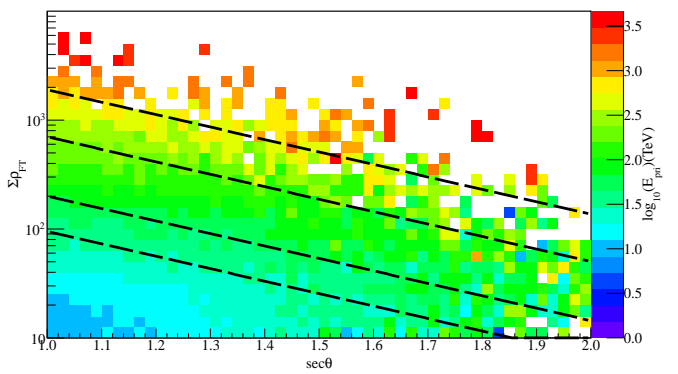

FIG. 1. - Simulated distribution of the log-mean energy of primary CRs as a function of $\sum \rho_{F T}$ and zenith angle. The $y$-axis is $\sum \rho_{F T}$; the $x$-axis is sec $\theta$, where $\theta$ is the reconstructed zenith angle; and the color scale represents the reconstructed log-mean energy in units of TeV. Dashed lines mark out the borders of events with different energies $(15,50,100,300$, and $1000 \mathrm{TeV})$.

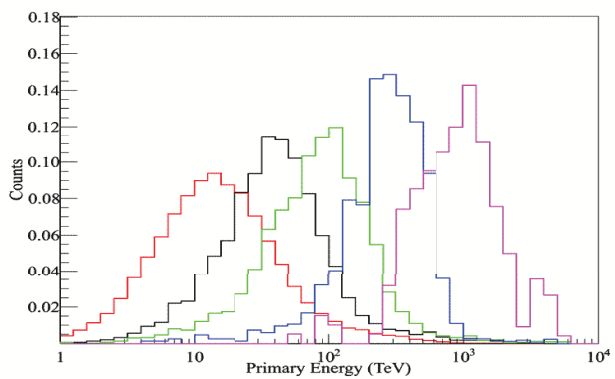

FIG. 2.- Normalized event number as a function of the simulated primary energy in each of the five energy samples, based on MC data. The log-mean energies of the five samples are $15 \mathrm{TeV}$ (red), $50 \mathrm{TeV}$ (black), $100 \mathrm{TeV}$ (green), $300 \mathrm{TeV}$ (blue), and $1 \mathrm{PeV}$ (pink).

$(15 \mathrm{TeV}), 3.97 \times 10^{9}(50 \mathrm{TeV}), 1.96 \times 10^{9}(100 \mathrm{TeV})$, $2.71 \times 10^{8}(300 \mathrm{TeV})$, and $5.72 \times 10^{7}(1 \mathrm{PeV})$, as listed in Table 1 .

\subsection{Analysis of the first harmonics of the anisotropy}

We employ the All-Distance Equi-Zenith Angle Method (Amenomori et al. 2005a, 2006), which has been shown to be sensitive to probe the large-scale anisotropy, to analyze the data. Details about this method can be found in (Amenomori et al. 2005a). The sky of the horizontal coordinate is divided into cells with a bin size of $1^{\circ}$ in both zenith (from $0^{\circ}$ to $60^{\circ}$ ) and azimuth (from $0^{\circ}$ to $\left.360^{\circ}\right)$. For the equatorial coordinate, the sky is divided into cells of $2^{\circ} \times 2^{\circ}$ between $0^{\circ}$ and $360^{\circ}$ in the R.A. $(\alpha)$ and between $-30^{\circ}$ and $90^{\circ}$ in the decl. $(\delta)$. The two-dimensional (2D) map in the equatorial coordinate is then smoothed in a window, changing the window widths between 5 and $30^{\circ}$.

To quantify the magnitude of the anisotropy, we project the two-dimensional (2D) anisotropy map before smoothing onto the R.A. axis, through averaging the relative intensities in all declinations from $-30^{\circ}$ to $90^{\circ}$, to derive the one-dimensional (1D) profile of the anisotropy. The R.A. is binned into 18 bins for this $1 \mathrm{D}$ analysis, and the $1 \mathrm{D}$ profile of the anisotropy is fitted by the first order harmonic function in form of

$$
R(\alpha)=1+A_{1} \cos \left(\alpha-\phi_{1}\right),
$$

where $R(\alpha)$ denotes the relative intensity of CRs at R.A. $\alpha, A_{1}$ is the amplitude of the first harmonics, and $\phi_{1}$ is the phase at which $R(\alpha)$ reaches its maximum.

\section{RESULTS}

\subsection{Sidereal Anisotropy map at $300 \mathrm{TeV}$}

Figure 3 shows the significance map and the relative intensity map of the sidereal anisotropy for the $300 \mathrm{TeV}$ energy sample. A smoothing with an optimized window width of $30^{\circ}$ is applied in this figure. We combined the $300 \mathrm{TeV}$ and $1 \mathrm{PeV}$ samples together in this figure to increase the statistics. The total event number used in this figure is $3.28 \times 10^{8}$, and the median energy is approximately $300 \mathrm{TeV}$.

From the significance map, we find that two regions are significant-that is, an excess centered at $\left(\alpha=263^{\circ}\right.$, $\delta=11^{\circ}$ ) with a significance of $7.2 \sigma$ and a deficit centered at $\left(\alpha=93^{\circ}, \delta=-25^{\circ}\right)$ with a significance of $-5.8 \sigma$. Note that the significance values are the pretrial results. We conservatively estimate a trial factor by assuming that all scans give statistically independent results. Since the search for this excess is performed over about $60 \times 180$ cells, and 26 different smoothing radii, the total trial factor is estimated to be about $2.81 \times 10^{5}$. The post-trial significance of the excess is $\sim 5.2 \sigma$. The deficit is no longer significant, fail to reach the $5 \sigma$ level, after the correction for the trials.

Because the acceptance of the detector decreases for larger zenith angles, the relative intensity map is similar but not completely the same as the significance map. An excess region centered at $\left(\alpha=269^{\circ}, \delta=-13^{\circ}\right)$ with a maximum excess of $+1.38 \times 10^{-3}$, and a deficit region centered at $\left(\alpha=87^{\circ}, \delta=-29^{\circ}\right)$ with a maximum deficit of $-1.80 \times 10^{-3}$ can be seen. Both the excess and deficit regions are consistent with the results of IceCube at $400 \mathrm{TeV}$ in the southern hemisphere (Abbasi et al. 2012). Combining these results gives us a full-sky picture of the sidereal anisotropy of GCRs at hundreds of $\mathrm{TeV}$.

The bottom panel in Figure 3 also shows the 1D projection of the relative intensity before the smoothing onto the R.A. axis. The correlation among different bins is carefully considered when calculating the statistical errors and fitting the data with the harmonic function in equation 1. If the correlation is not considered correctly, the errors of the fitting parameters would be underestimated. The blue curve shows the best-fitting result, with the fitting parameters indicated in the figure. The significance of non-zero amplitude is $5.6 \sigma$, which shows that the obtained first harmonics are indeed significant. The reduced $\chi^{2}$ value is $26.7 / 16$, which means that the first harmonic function can describe the $1 \mathrm{D}$ projected profile well.

One of the possible origins of the sidereal anisotropy is the Compton-Getting (CG) effect, due to the orbital motion of the solar system around the Galactic center (Compton \& Getting 1935). The relative intensity of this effect is carefully calculated by the $\mathrm{MC}$ method. Considering the location of the Tibet AS array $\left(90.522^{\circ} \mathrm{E}, 30.102^{\circ} \mathrm{N}\right)$, the velocity $\left(220 \mathrm{~km} \mathrm{~s}^{-1}\right)$ of the orbital motion of the solar system around the Galactic center and the spectrum index (2.7) of the CRs energy spectrum, the intensity of the sky that is divided into cells of $2^{\circ} \times 2^{\circ}$ between $0^{\circ}$ and $360^{\circ}$ in the R.A. $(\alpha)$ and between $-30^{\circ}$ and $90^{\circ}$ in the decl. $(\delta)$ is calculated. Then the identical analyses are performed to this $\mathrm{MC}$ data sample. 
The $\mathrm{R}(\alpha)$ expected from this $\mathrm{CG}$ effect, shown as the black dashed line in Figure 3 , has a maximum at $\left(\alpha=315^{\circ}, \delta=0^{\circ}\right)$ and a minimum at $\left(\alpha=135^{\circ}\right.$, $\delta=0^{\circ}$ ). Neither the amplitude nor the phase of the large-scale anisotropy observed in this work can be described in terms of the CG effect.

\subsection{Variation of $C R$ sidereal anisotropy with the energy between 10 and $1000 \mathrm{Te} V$}

Figure 4 shows the variation of the sidereal anisotropy with the energy between $10 \mathrm{TeV}$ and $1 \mathrm{PeV}$. At 15 $\mathrm{TeV}$ and $50 \mathrm{TeV}$, the tail-in and loss-cone features (Amenomori et al. 2006) are observed with very high significances. An intensity excess in the Cygnus region can also be seen. However, these features become less significant above $100 \mathrm{TeV}$, being replaced with some new features. At $300 \mathrm{TeV}$ and $1 \mathrm{PeV}$, the anisotropy maps are distinctly different from those in $15-50 \mathrm{TeV}$. We can clearly see the phase of the $1 \mathrm{D}$ projection changing with the primary energies, as seen in Table 11, showing the best-fit parameters.

Figure 5 compares the amplitude and phase obtained in this work with those reported so far from the deep underground muon experiments and extensive AS experiments. Our results are in close agreement with other results in similar energy regions in both the amplitude and the phase. It is interesting to note that a sharp increase of the amplitude above $100 \mathrm{TeV}$ can be seen in the upper panel. The origins of this feature cannot be explained with the conventional diffusion scenario of GCRs, and may provide us with a new hint for understanding the origin and propagation of GCRs.

\subsection{Anisotropy in Solar Time and Antisidereal Time}

In order to confirm that the obtained anisotropy is not affected by the seasonal variation of the AS array performance, identical analyses are performed in the solar time and antisidereal time frames in five energy samples. Figure 6] shows the local solar time and antisidereal time daily variations measured by Tibet AS Array in five energy samples, and the best-fit parameters are also shown in Table 1. The amplitude and phase in the solar time frame are in good agreement with the expectation from the $\mathrm{CG}$ effect due to the terrestrial orbital motion around the Sun $\left(A_{\mathrm{sol}, \mathrm{CG}}=0.047 \%\right.$ and $\left.\phi_{\mathrm{sol}, \mathrm{CG}}=6.0 \mathrm{hr}\right)$. In all five energy samples, no significant anisotropy is observed in the antisidereal time frame, ensuring that no additional correction is required for the seasonal effects. The observed results in the solar and antisidereal time frames support the reliability of the observed sidereal anisotropy.

\section{CONCLUSION AND DISCUSSION}

Fifteen years data recorded by the Tibet AS array have been analyzed to study the sidereal anisotropy of CRs. In this work, we improve the estimate of the primary CR energies through a $2 \mathrm{D}$ cut in the $\sum \rho_{F T}-\sec \theta$ plane, to explore the anisotropy including larger zenith angle events. For the first time, we extend the analyzed decl. down to $-30^{\circ}$ to complete a full-sky coverage of the anisotropy at hundreds of $\mathrm{TeV}$ energies by combining with the IceCube's results at the South Pole. The 2D anisotropy map at $\sim 300 \mathrm{TeV}$ obtained in this work is smoothly connected with IceCube's results at $400 \mathrm{TeV}$. The energy dependence of the large-scale sidereal anisotropy has been derived between $10 \mathrm{TeV}$ and $1 \mathrm{PeV}$. We measured the energy dependence of the first harmonics of the anisotropy above $100 \mathrm{TeV}$, which may be associated with local origins of GCRs.

The CG effect expected from the orbital motion of the solar system around the Galatic center is not observed at $300 \mathrm{TeV}$, as shown in Figure 3. The basic picture that GCRs are co-rotating with the local Galactic neighbors still holds at this energy Amenomori et al. 2006). As pointed out earlier, the GCR rest frame may have a smaller relative velocity with a different direction from neighboring stars and the interstellar medium (Abbasi et al. 2012). This scenario is possibly responsible for the GCR anisotropy observed at hundreds of $\mathrm{TeV}$.

The strongest excesses at hundreds of $\mathrm{TeV}$ are from the direction of the Galactic center, which may imply a Galactic center origin of GCRs at these energies (Guo et al. 2013). It is interesting to note that the highest-energy $\mathrm{CR}$ accelerators have been identified by the HESS telescope in the Galactic center (HESS Collaboration et al. 2016). However, the energy dependences of the amplitude and phase cannot be easily understood in a simple diffusion scenario with any types of GCR sources.

The sharp increase of the amplitude above $100 \mathrm{TeV}$ may imply an evolution of propagation parameters, such as spatial parameters (Tomassetti 2015; Guo et al. 2016). The knowledge of the propagation of GCRs needs to be further improved for our full understanding the properties of the anisotropy, especially in this high-energy region where the conventional diffusion/drift models may not work any more. Finally, we add to note that the measurements of the anisotropy above $\mathrm{PeV}$, which is possibly associated with the knee of GCRs, are very important to advance our understanding of origin and propagation of GCRs.

\section{ACKNOWLEDGMENTS}

The collaborative experiment of the Tibet Air Shower Arrays has been performed under the auspices of the Ministry of Science and Technology of China and the Ministry of Foreign Affairs of Japan. This work was supported in part by a Grant-in-Aid for Scientific Research on Priority Areas from the Ministry of Education, Culture, Sports, Science and Technology, by Grants-inAid for Science Research from the Japan Society for the Promotion of Science in Japan, and by the Grants from the National Natural Science Foundation of China and the Chinese Academy of Sciences. Zhaoyang Feng is supported by the Natural Sciences Foundation of China (Nos.11405182, Nos.1135010). C. Liu is supported by the Natural Sciences Foundation of China (Nos. 11405180).

\section{REFERENCES}

Aartsen, M. G., Abbasi, R., Abdou, Y., et al. 2013, ApJ, 765, 55

Abbasi, R., Abdou, Y., Abu-Zayyad, T., et al. 2010, ApJ, 718 , L194
—. 2012, ApJ, 746, 33

Abdo, A. A., Allen, B. T., Aune, T., et al. 2009, ApJ, 698, 2121 
Aglietta, M., Alessandro, B., Antonioli, P., et al. 1995, International Cosmic Ray Conference, 2, 800

-. 1996, ApJ, 470, 501

Aglietta, M., Alekseenko, V. V., Alessandro, B., et al. 2009, ApJ, 692, L130

Ahlers, M., \& Mertsch, P. 2016, ArXiv e-prints, arXiv:1612.01873

Alekseenko, V. V., Cherniaev, A. B., Djappuev, D. D., et al. 2009, Nuclear Physics B Proceedings Supplements, 196, 179

Alexeyenko, V. V., Chudakov, A. E., Gulieva, E. N., \& Sborschikov, V. G. 1981, International Cosmic Ray Conference, 2,146

Ambrosio, M., Antolini, R., Baldini, A., et al. 2003, Phys. Rev. D, 67,042002

Amenomori, M., Ayabe, S., Cui, S. W., et al. 2003, ApJ, 598, 242

Amenomori, M., Ayabe, S., Chen, D., et al. 2005a, ApJ, 633, 1005

Amenomori, M., Ayabe, S., Cui, S. W., et al. 2005b, ApJ, 626 , L29

Amenomori, M., Ayabe, S., Bi, X. J., et al. 2006, Science, 314, 439

Amenomori, M., Bi, X. J., Chen, D., et al. 2013, Proceedings of the $33^{r d}$ ICRC, Rio de Janeiro, ID256

-. 2015, Proceedings of the $34^{\text {th }}$ ICRC, the Haag, ID355

Andreyev, Y. M., Chudakov, A. E., Kozyarivsky, V. A., et al. 1987, International Cosmic Ray Conference, 2, 22

Antoni, T., Apel, W. D., Badea, A. F., et al. 2004, ApJ, 604, 687

Bartoli, B., Bernardini, P., Bi, X. J., et al. 2015, ApJ, 809, 90

Bercovitch, M., \& Agrawal, S. P. 1981, International Cosmic Ray Conference, 10, 246

Compton, A. H., \& Getting, I. A. 1935, Physical Review, 47, 817

Cutler, D. J., \& Groom, D. E. 1991, ApJ, 376, 322

Feng, Z., Zhang, Y., Liu, C., et al. 2009, Proceedings of the $31^{\text {st }}$ ICRC, Łódź, ID0869

Fenton, K. B., Fenton, A. G., \& Humble, J. E. 1995, International Cosmic Ray Conference, 4, 635

Gombosi, T., Kóta, J., Somogyi, A. J., et al. 1975, International Cosmic Ray Conference, 2, 586
Guillian, G., Hosaka, J., Ishihara, K., et al. 2007, Phys. Rev. D, 75,062003

Guo, Y.-Q., Feng, Z.-Y., Yuan, Q., Liu, C., \& Hu, H.-B. 2013, New Journal of Physics, 15, 013053

Guo, Y.-Q., Tian, Z., \& Jin, C. 2016, ApJ, 819, 54

Heck, D., Knapp, J., Capdevielle, J. N., Schatz, G., \& Thouw, T. 1998, CORSIKA: a Monte Carlo code to simulate extensive air showers.

HESS Collaboration, Abramowski, A., Aharonian, F., et al. 2016, Nature, 531, 476

Hörandel, J. R. 2003, Astroparticle Physics, 19, 193

Kasahara, K. 2006,

http://cosmos.n.kanagawa-u.ac.jp/EPICSHome/index.html

Kifune, T., Hara, T., Hatano, Y., et al. 1986, Journal of Physics G Nuclear Physics, 12, 129

Lee, Y. W., \& Ng, L. K. 1987, International Cosmic Ray Conference, 2, 18

Mori, S., Yasue, S., Munakata, K., et al. 1995, International Cosmic Ray Conference, 4, 648

Moskalenko, I. V., Strong, A. W., Ormes, J. F., \& Potgieter, M. S. 2002, ApJ, 565, 280

Munakata, K., Yasue, S., Mori, S., et al. 1995, International Cosmic Ray Conference, 4, 639

Munakata, K., Kiuchi, T., Yasue, S., et al. 1997, Phys. Rev. D, 56,23

Nagashima, K., Fujimoto, K., Sakakibara, S., Fujii, Z., \& Ueno, H. 1989, Nuovo Cimento C Geophysics Space Physics C, 12, 695

Sakakibara, S., Ueno, H., Fujimoto, K., Kondo, I., \& Nagashima, K. 1973, International Cosmic Ray Conference, 2, 1058

Swinson, D. B., \& Nagashima, K. 1985, Planet. Space Sci., 33, 1069

Thambyahpillai, T. 1983, International Cosmic Ray Conference, 3,383

Tomassetti, N. 2015, Phys. Rev. D, 92, 081301

Ueno, H., Fujii, Z., \& Yamada, T. 1990, International Cosmic Ray Conference, 6, 361
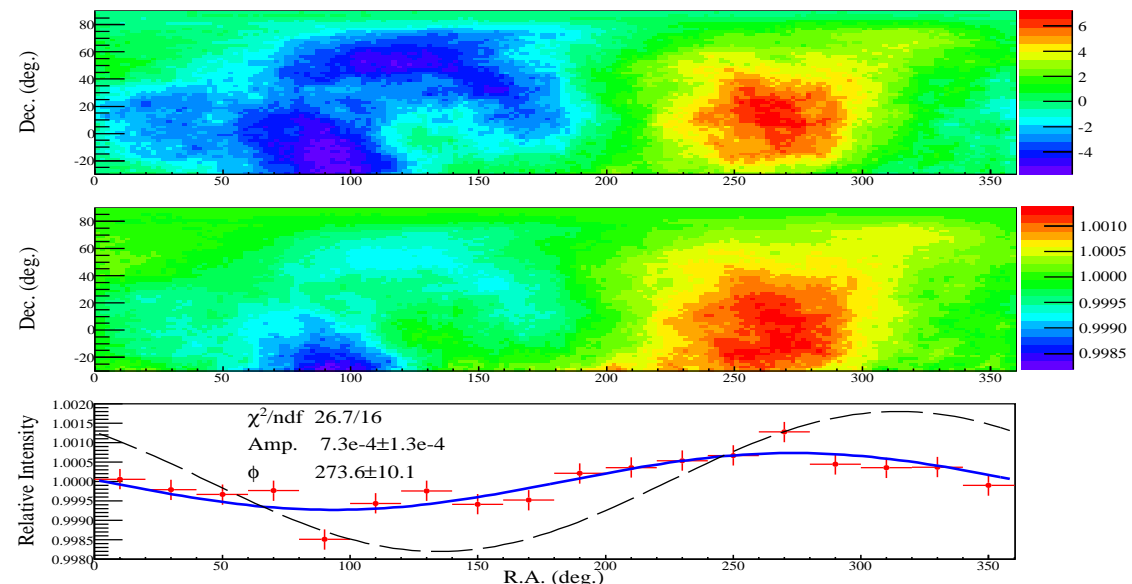

Fig. 3. - Large-scale sidereal anisotropy at $300 \mathrm{TeV}$ by the Tibet AS Array. The 2D maps are smoothed with a $30^{\circ}$ Gaussian kernel. The top and middle panels display the significance and relative intensity maps, respectively, while the bottom one shows the 1D projection of the 2D map onto the R.A. axis. The blue curve shows the first harmonic fitting to the data, and the black dashed line is the predicted Galactic CG effect with an amplitude of $\sim 0.19 \%$.

TABLE 1

Fitting Results of the first harmonic (Amplitude, Phase, and Reduced $\chi^{2}$ ) in the sidereal (Columns 2-4), solar (Columns 5-7), and Antisidereal (Columns 8-10) Times. The number of events in each energy sample is given in Column 11.

\begin{tabular}{|c|c|c|c|c|c|c|c|c|c|c|}
\hline $\begin{array}{c}\text { Energy } \\
\text { TeV }\end{array}$ & $\begin{array}{c}A_{\text {sid }} \\
10^{-4}\end{array}$ & $\begin{array}{c}\phi_{\text {sid }} \\
\left.{ }^{\circ}\right]\end{array}$ & $\chi_{s i d}^{2} / n d f$ & $\begin{array}{c}A_{\text {sol }} \\
10^{-4}\end{array}$ & $\begin{array}{c}\phi_{\text {sol }} \\
\mathrm{hr}\end{array}$ & $\overline{\chi_{\text {sol }}^{2} / n d f}$ & $\begin{array}{c}A_{\text {asid }} \\
10^{-4}\end{array}$ & $\begin{array}{c}\phi_{\text {asid }} \\
\mathrm{hr}\end{array}$ & $\chi_{\text {asid }}^{2} / n d f$ & Number of Event \\
\hline 50 & $5.3 \pm 0.4$ & $20.8 \pm 4.7$ & $152.9 / 16$ & $4.6 \pm 0.4$ & $6.37 \pm 0.35$ & $46.7 / 16$ & $0.39 \pm 0.43$ & $22.5 \pm 4.2$ & $46.7 / 16$ & $3.97 \times 10^{9}$ \\
\hline 100 & $2.7 \pm 0.6$ & $326.8 \pm 12.0$ & $67.6 / 16$ & $4.0 \pm 0.6$ & $5.91 \pm 0.53$ & $14.2 / 16$ & $0.80 \pm 0.56$ & $22.3 \pm 2.7$ & $9.8 / 16$ & $1.96 \times 10^{9}$ \\
\hline 1000 & $13.0 \pm 3.0$ & $286.6 \pm 12.6$ & $9.3 / 16$ & $9.8 \pm 2.8$ & $6.97 \pm 1.13$ & $10.9 / 16$ & $1.0 \pm 2.9$ & $14.8 \pm 11.0$ & $6.3 / 16$ & $5.72 \times 10^{7}$ \\
\hline
\end{tabular}



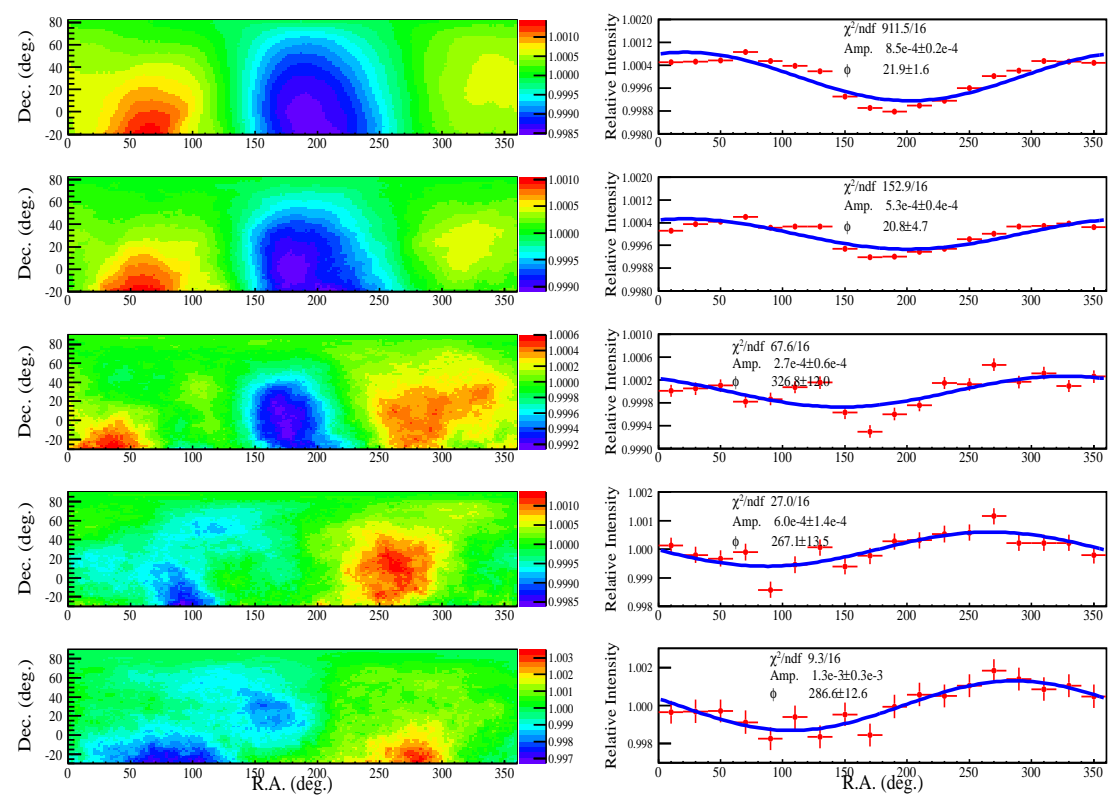

FIG. 4.-2D anisotropy maps in five energy samples $(15,50,100,300$, and $1000 \mathrm{TeV}$, from top to bottom). Left panels show the relative intensity maps (with $30^{\circ}$ smoothing), while right panels show the $1 \mathrm{D}$ projections. The meaning of the blue curves in the right panels is the same as in Figure 3
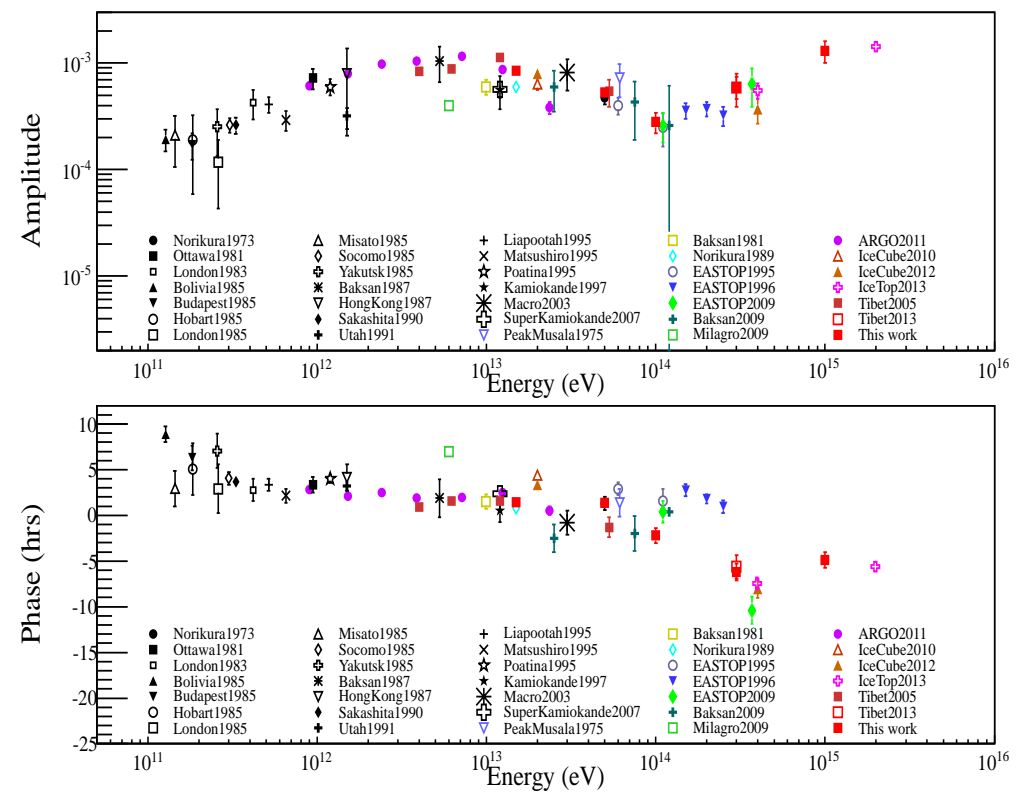

FIG. 5. - The energy dependences of amplitude (top) and phase (bottom) of the first harmonics of the CRs anisotropy obtained in this work, and reported from previous measurements. They are underground muon observations: Norikura(1973) (Sakakibara et al. 1973), Ottawa(1983) (Bercovitch \& Agrawal 1981), London(1983) (Thambvahpillai 1983), Bolivia(1985) (Swinson \& Nagashima 1985), Budapest(1985) (Swinson \& Nagashima 1985), Hobart(1985) (Swinson \& Nagashima 1985), London(1985) (Swinson \& Nagashima 1985), Misato(1985) (Swinson \& Nagashima 1985), Socorro(1985) (Swinson \& Nagashima 1985), Yakutsk(1985) (Swinson \& Nagashima 1985), Banksan(1987) (Andreyev et al. 1987), HongKong(1987) (Lee \& Ng 1987), Sakashita(1990) (Ueno et al. 1990), Utah(1991) (Cutler \& Groom 1991), Liapootah(1995) (Munakata et al. 1995), Matsushiro(1995) (Mori et al. 1995), Poatina(1995) (Fenton et al. 1995), Kamiokande(1997) (Munakata et al. 1997), Marco(2003) (Ambrosio et al. 2003), SuperKamiokande(2007) (Guillian et al. 2007), and air shower array experiments: PeakMusala(1975) (Gombosi et al. 1975), Baksan(1981) (Alexeyenko et al. 1981), Norikura(1989) (Nagashima et al. 1989), EASTOP(1995,1996,2009) (Aglietta et al. 1995, 1996, 2009), Baksan(2009) (Alekseenko et al. 2009), Milagro(2009) (Abdo et al. 2009), IceCube(2010,2012) (Abbasi et al. 2010, 2012), IceTop(2013) (Aartsen et al. 2013), ARGO-YBJ(2015) (Bartoli et al. 2015), Tibet(2005,2013) (Amenomori et al.|2005b; Amenomori et al. 2013). 

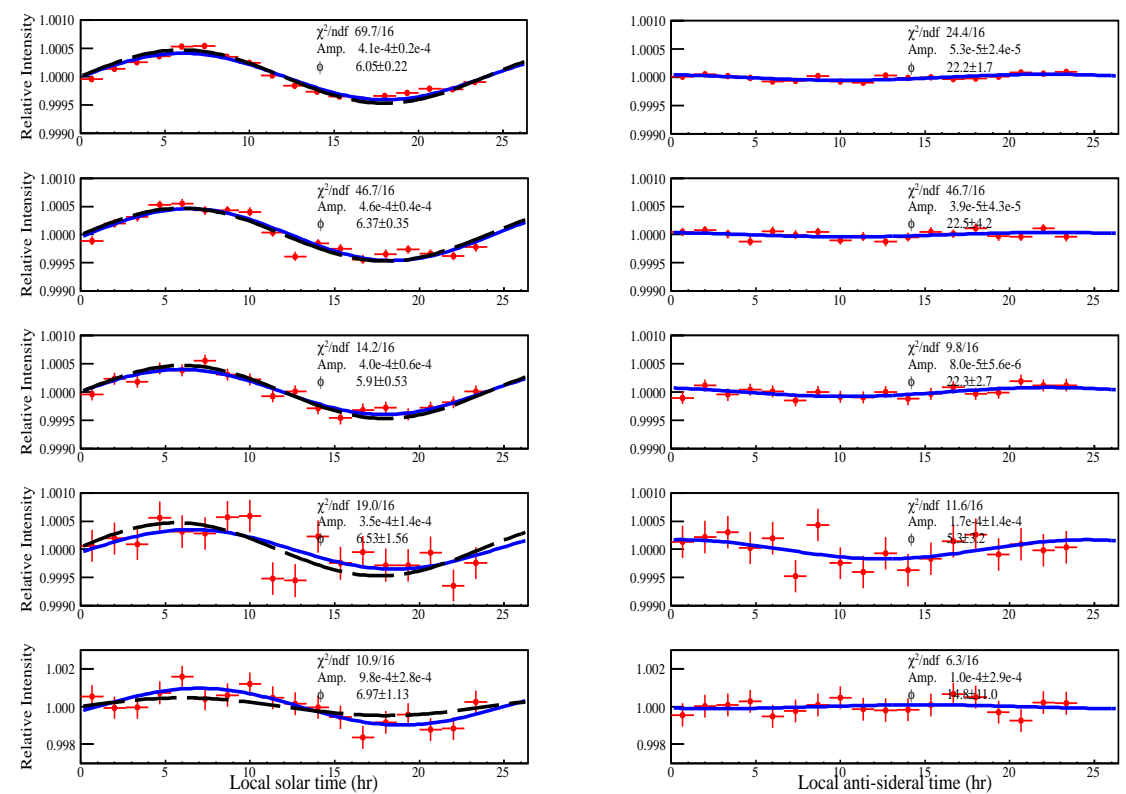

FIG. 6. - Local solar time and antisidereal time daily variations measured by Tibet AS Array in five energy samples, 15, 50 , 100, 300, and $1000 \mathrm{TeV}$, from top to bottom. Left panels show the solar time daily variations, with blue curves showing the first harmonic fit to the data and the black dashed curves indicating the expected CG effect due to the Earth's orbital motion around the sun, with an amplitude of $0.047 \%$ and an phase of $6 \mathrm{hr}$. Right panels show the antisidereal time daily variations and the first harmonic fitting results. 